Article

\title{
Nonlinear Gronwall-Bellman Type Inequalities and Their Applications
}

\author{
Weimin Wang ${ }^{1}$, Yuqiang Feng ${ }^{2, *}$ and Yuanyuan Wang ${ }^{1}$ \\ 1 School of Science, Wuhan University of Science and Technology, Wuhan 430065, China; \\ wugongren@163.com (W.W.); wangyuanyuan@wust.edu.cn (Y.W.) \\ 2 Hubei Province Key Laboratory of Systems Science in Metallurgical Process, Wuhan 430065, China \\ * Correspondence: yqfeng6@126.com; Tel.: +86-027-6889-3262
}

Academic Editor: Hari M. Srivastava

Received: 2 February 2017; Accepted: 23 May 2017; Published: 31 May 2017

\begin{abstract}
In this paper, some nonlinear Gronwall-Bellman type inequalities are established. Then, the obtained results are applied to study the Hyers-Ulam stability of a fractional differential equation and the boundedness of solutions to an integral equation, respectively.
\end{abstract}

Keywords: nonlinear Gronwall-Bellman inequalities; differential equation; integral equation; Hyers-Ulam stability; boundedness

\section{Introduction}

The study of Gronwall-Bellman inequalities has been paid much attention and developed at a high rate in the last three decades. These inequalities play an important role in many fields. They are applied to the investigation of the stability, boundedness, global existence, uniqueness, and continuous dependence on the initial or boundary value and parameters of solutions to differential equations, integral equations, as well as difference equations [1-11]. They are also used to study the regularized family of models for homogeneous incompressible two-phase flows [12], the state of the high nonlinear circuit [13], the Cousin problems and the emergence of the Sheaf Concept [14].

Recently, Willett [15] discussed the linear inequality:

$$
u(t) \leq w_{0}(t)+\sum_{i=1}^{n} w_{i}(t) \int_{0}^{t} v_{i}(s) u(s) \mathrm{d} s \quad(t \in I)
$$

and Lin [16] extended the study to the linear inequality as follows:

$$
u(t) \leq a(t)+\sum_{i=1}^{n} b_{i}(t) \int_{0}^{t}(t-s)^{\beta_{i}-1} u(s) \mathrm{d} s
$$

Several generalizations of the Gronwall inequality were established and then applied to prove the uniqueness of solutions for fractional differential equations with various derivatives.

In this paper, we are concerned with the following nonlinear Gronwall-Bellman-type inequality:

$$
\begin{aligned}
u^{p}(x) \leq & a(x)+\sum_{i=1}^{n} \omega_{i}(x) \int_{0}^{x} h_{i}(t) g_{i}(t, u(t)) \mathrm{d} t \\
& +\sum_{i=1}^{n} b_{i}(x) \int_{0}^{x}(x-t)^{\beta_{i}-1} f_{i}(t, u(t)) \mathrm{d} t
\end{aligned}
$$


Some new results are obtained and then applied to investigate the qualitative properties of differential and integral equations.

This paper is organized as follows: In Section 2, we introduce some definitions and notations. Some nonlinear Gronwall-Bellman-type inequalities are presented in Section 3. In Section 4, the obtained results are applied to prove the Hyers-Ulam stability of a fractional differential equation and the boundedness of the solutions to a integral equation.

\section{Preliminaries}

In this section, we recall some standard definitions and notations.

Lemma 1. (see [17]). Assume that $a \geq 0, p \geq q \geq 0$ with $p \neq 0$. Then, for any $b>0$, we have:

$$
a^{\frac{q}{p}} \leq \frac{q}{p} b^{\frac{q-p}{p}} a+\frac{p-q}{p} b^{\frac{q}{p}}
$$

Lemma 2. (see [15]) Suppose that:

$$
u(t) \leq w_{0}(t)+\sum_{i=1}^{n} w_{i}(t) \int_{0}^{t} v_{i}(s) u(s) \mathrm{d} s \quad(t \in I)
$$

where $v_{i} w_{j}(i=1,2 \cdots, n ; j=0,1, \cdots, n)$ and $v_{i} u(i=1,2, \cdots, n)$ are locally integrable on $I$, all functions are assumed non-negative. Then:

$$
u \leq E_{n} w_{0}
$$

where $E_{i}(i=0,1, \cdots, n)$ is defined inductively as the composition of $i+1$ functional operators; i.e., $E_{i}=D_{i} D_{i-1} \cdots D_{0}$, where:

$$
\begin{aligned}
& D_{0} w=w \\
& D_{j} w=w+\left(E_{j-1} w_{j}\right)\left(\exp \int_{0}^{t} v_{j} E_{j-1} w_{j}\right) \int_{0}^{t} v_{j} w \mathrm{~d} s \quad(j=1,2, \cdots, n)
\end{aligned}
$$

\section{Main Results}

In this section, we will establish some nonlinear Gronwall-Bellman-type inequalities. The first result is the following:

Theorem 1. Suppose that $p \geq 1, \beta_{i}>0$ are constants, $f_{i} \in C\left(R_{+} \times R_{+}, R_{+}\right)$with $0 \leq f_{i}(x, u)-f_{i}(x, v) \leq$ $l_{i}(u-v)$ for $u \geq v \geq 0$, where $l_{i}$ is the Lipschitz constants, and all the functions are nonnegative and continuous on $[0, T)$, with $b_{i}(i=1,2, \cdots, n)$ are bounded and nondecreasing functions. If the following inequality is satisfied:

$$
u^{p}(x) \leq a(x)+\sum_{i=1}^{n} b_{i}(x) \int_{0}^{x}(x-t)^{\beta_{i}-1} f_{i}(t, u(t)) \mathrm{d} t
$$

then:

$$
\begin{aligned}
u(x) \leq & \left(\tilde{a}(x)+\sum_{k=1}^{\infty}\left(\sum_{1^{\prime}, 2^{\prime}, \ldots k^{\prime}=1}^{n}\left(\frac{1}{p} b^{\frac{1-p}{p}}\right)^{k} \cdot \frac{\prod_{i=1}^{k}\left[l_{i^{\prime}} b_{i^{\prime}}(x) \Gamma\left(\beta_{i^{\prime}}\right)\right]}{\Gamma\left(\sum_{i=1}^{k} \beta_{i^{\prime}}\right)} .\right.\right. \\
& \left.\left.\int_{0}^{x}(x-t)^{\sum_{i=1}^{k} \beta_{i^{\prime}}-1} \tilde{a}(t) \mathrm{d} t\right)\right)^{\frac{1}{p}}
\end{aligned}
$$

for any $b>0$ and $t \in[0, T]$, where:

$$
\tilde{a}(x)=a(x)+\sum_{i=1}^{n} b_{i}(x) \int_{0}^{x}(x-t)^{\beta_{i}-1} f_{i}\left(t, \frac{p-1}{p} b^{\frac{1}{p}}\right) \mathrm{d} t
$$


Proof. Denote $u^{p}(x)=v(x)$, then we have $u(x)=v^{\frac{1}{p}}(x)$ :

$$
v(x) \leq a(x)+\sum_{i=1}^{n} b_{i}(x) \int_{0}^{x}(x-t)^{\beta_{i}-1} f_{i}\left(t, v^{\frac{1}{p}}(t)\right) \mathrm{d} t
$$

According to Lemma 1:

$$
\begin{aligned}
v(x) \leq & a(x)+\sum_{i=1}^{n} b_{i}(x) \int_{0}^{x}(x-t)^{\beta_{i}-1} f_{i}\left(t, \frac{1}{p} b^{\frac{1-p}{p}} v(t)+\frac{p-1}{p} b^{\frac{1}{p}}\right) \mathrm{d} t \\
& =a(x)+\sum_{i=1}^{n} b_{i}(x) \int_{0}^{x}(x-t)^{\beta_{i}-1}\left[f_{i}\left(t, \frac{1}{p} b^{\frac{1-p}{p}} v(t)+\frac{p-1}{p} b^{\frac{1}{p}}\right)\right. \\
& \left.-f_{i}\left(t, \frac{p-1}{p} b^{\frac{1}{p}}\right)+f_{i}\left(t, \frac{p-1}{p} b^{\frac{1}{p}}\right)\right] \mathrm{d} t \\
\leq & a(x)+\frac{1}{p} b^{\frac{1-p}{p}} \sum_{i=1}^{n} l_{i} b_{i}(x) \int_{0}^{x}(x-t)^{\beta_{i}-1} v(t) \mathrm{d} t \\
& +\sum_{i=1}^{n} b_{i}(x) \int_{0}^{x}(x-t)^{\beta_{i}-1} f_{i}\left(t, \frac{p-1}{p} b^{\frac{1}{p}}\right) \mathrm{d} t \\
= & \tilde{a}(x)+\frac{1}{p} b^{\frac{1-p}{p}} \sum_{i=1}^{n} l_{i} b_{i}(x) \int_{0}^{x}(x-t)^{\beta_{i}-1} v(t) \mathrm{d} t
\end{aligned}
$$

Now let:

$$
B \varphi(x)=\sum_{i=1}^{n} \frac{1}{p} b^{\frac{1-p}{p}} l_{i} b_{i}(x) \int_{0}^{x}(x-t)^{\beta_{i}-1} \varphi(t) \mathrm{d} t
$$

$x \in[0, T)$, for locally integrable functions $\varphi$. Then:

$$
v(x) \leq \tilde{a}(x)+B v(x) .
$$

By mathematical induction method, we have:

$$
\begin{aligned}
v(x) & \leq \tilde{a}(x)+B(\tilde{a}(x)+B v(x)) \\
& =\tilde{a}(x)+B \tilde{a}(x)+B^{2} v(x) \\
& \leq \tilde{a}(x)+B \tilde{a}(x)+B^{2}(\tilde{a}(x)+B v(x)) \\
& =\tilde{a}(x)+B \tilde{a}(x)+B^{2} \tilde{a}(x)+B^{3} v(x) \\
& \leq \cdots \\
& \leq \sum_{m=0}^{k-1} B^{m} \tilde{a}(x)+B^{k} v(x) .
\end{aligned}
$$

Let $r=\frac{1}{p} b^{\frac{1-p}{p}}$. We assert that:

$$
B^{k} v(x) \leq \sum_{1^{\prime}, 2^{\prime}, \cdots k^{\prime}=1}^{n} r^{k} \frac{\prod_{i=1}^{k}\left[l_{i^{\prime}} b_{i^{\prime}}(x) \Gamma\left(\beta_{i^{\prime}}\right)\right]}{\Gamma\left(\sum_{i=1}^{k} \beta_{i^{\prime}}\right)} \int_{0}^{x}(x-t)^{\sum_{i=1}^{k} \beta_{i^{\prime}}-1} v(t) \mathrm{d} t
$$

for any $k \in N$ and $B^{k} v(x) \rightarrow 0$ as $k \rightarrow \infty$ for each $x$ in $0 \leq x<T$. 
In fact, (i) If $k=1$, then:

$$
\begin{aligned}
B v(x) & \leq \sum_{1^{\prime}=1}^{n} r \frac{\left[l_{i^{\prime}} b_{i^{\prime}}(x) \Gamma\left(\beta_{i^{\prime}}\right)\right]}{\Gamma\left(\beta_{i^{\prime}}\right)} \int_{0}^{x}(x-t)^{\beta_{i^{\prime}}-1} v(t) \mathrm{d} t \\
& =\sum_{1^{\prime}=1}^{n} r l_{i^{\prime}} b_{i^{\prime}}(x) \int_{0}^{x}(x-t)^{\beta_{i^{\prime}}-1} v(t) \mathrm{d} t \\
& =B v(x)
\end{aligned}
$$

So we know the inequality (17) holds for $k=1$.

(ii) Assume that the inequality (17) holds for $k=j$; that is:

$$
B^{j} v(x) \leq \sum_{1^{\prime}, 2^{\prime}, \cdots j^{\prime}=1}^{n} r^{j} \frac{\prod_{i=1}^{j}\left[l_{i^{\prime}} b_{i^{\prime}}(x) \Gamma\left(\beta_{i^{\prime}}\right)\right]}{\Gamma\left(\sum_{i=1}^{j} \beta_{i^{\prime}}\right)} \int_{0}^{x}(x-t)^{\sum_{i=1}^{j} \beta_{i^{\prime}}-1} v(t) \mathrm{d} t
$$

(iii) For $k=j+1$, we have:

$$
\begin{aligned}
B^{j+1} v(x) & =B\left(B^{j} v(x)\right) \\
& \leq \sum_{i=1}^{n} r l_{i} b_{i}(x) \int_{0}^{x}(x-t)^{\beta_{i}-1} \cdot( \\
& \left.\sum_{1^{\prime}, 2^{\prime}, \ldots j^{\prime}=1}^{n} r^{j} \frac{\prod_{i=1}^{j}\left[l_{i^{\prime}} b_{i^{\prime}}(t) \Gamma\left(\beta_{i^{\prime}}\right)\right]}{\Gamma\left(\sum_{i=1}^{j} \beta_{i^{\prime}}\right)} \cdot \int_{0}^{t}(t-s)^{\sum_{i=1}^{j} \beta_{i^{\prime}}-1} v(s) \mathrm{d} s\right) \mathrm{d} t
\end{aligned}
$$

Owing to the monotonicity of $b_{i}(i=1,2, \cdots, n)$, we get:

$$
\begin{gathered}
B^{j+1} v(x) \leq \sum_{i=1}^{n} r l_{i} b_{i}(x) \cdot \sum_{1^{\prime}, 2^{\prime}, \ldots j^{\prime}=1}^{n} r^{j} \frac{\prod_{i=1}^{j}\left[l_{i^{\prime}} b_{i^{\prime}}(t) \Gamma\left(\beta_{i^{\prime}}\right)\right]}{\Gamma\left(\sum_{i=1}^{j} \beta_{i^{\prime}}\right)} . \\
\int_{0}^{x}(x-t)^{\beta_{i}-1} \cdot \int_{0}^{t}(t-s)^{\sum_{i=1}^{j} \beta_{i^{\prime}}-1} v(s) \mathrm{d} s \mathrm{~d} t
\end{gathered}
$$

Interchanging the order of integration, we have:

$$
\begin{aligned}
B^{j+1} v(x) \leq & r^{j+1} \sum_{i=1}^{n} l_{i} b_{i}(x) \cdot \sum_{1^{\prime}, 2^{\prime}, \ldots j^{\prime}=1}^{n} \frac{\prod_{i=1}^{j}\left[l_{i^{\prime}} b_{i^{\prime}}(x) \Gamma\left(\beta_{i^{\prime}}\right)\right]}{\Gamma\left(\sum_{i=1}^{j} \beta_{i^{\prime}}\right)} . \\
& \frac{\Gamma\left(\beta_{i}\right) \Gamma\left(\sum_{i=1}^{j} \beta_{i^{\prime}}\right)}{\Gamma\left(\beta_{i}+\sum_{i=1}^{j} \beta_{i^{\prime}}\right)} \cdot \int_{0}^{x}(x-s)^{\sum_{i=1}^{j} \beta_{i^{\prime}}+\beta_{i}-1} v(s) \mathrm{d} s \\
= & r^{j+1} \sum_{i=1}^{n} \sum_{1^{\prime}, 2^{\prime}, \ldots j^{\prime}=1}^{n} \frac{\left[l_{i} b_{i}(x) \Gamma\left(\beta_{i}\right)\right] \cdot \prod_{i=1}^{j}\left[l_{i^{\prime}} b_{i^{\prime}}(x) \Gamma\left(\beta_{i^{\prime}}\right)\right]}{\Gamma\left(\beta_{i}+\sum_{i=1}^{j} \beta_{i^{\prime}}\right)} . \\
& \int_{0}^{x}(x-s)^{\sum_{i=1}^{j} \beta_{i^{\prime}}+\beta_{i}-1} v(s) \mathrm{d} s
\end{aligned}
$$

Let $i=(j+1)^{\prime}$, then: 


$$
\begin{aligned}
B^{j+1} v(x) \leq & r^{j+1} \sum_{(j+1)^{\prime}=1}^{n} \sum_{1^{\prime}, 2^{\prime}, \ldots j^{\prime}=1}^{n} \frac{\left[l_{(j+1)^{\prime}} b_{(j+1)^{\prime}}(x) \Gamma\left(\beta_{(j+1)^{\prime}}\right)\right] \cdot \prod_{i=1}^{j}\left[b_{i^{\prime}}(x) \Gamma\left(\beta_{i^{\prime}}\right)\right]}{\Gamma\left(\beta_{(j+1)^{\prime}}+\sum_{i=1}^{j} \beta_{i^{\prime}}\right)} . \\
& \int_{0}^{x}(x-s)^{\sum_{i=1}^{j} \beta_{i^{\prime}}+\beta_{(j+1)^{\prime}}-1} v(s) \mathrm{d} s \\
= & r^{j+1} \sum_{1^{\prime}, 2^{\prime}, \ldots(j+1)^{\prime}=1}^{n} \frac{\prod_{i=1}^{j+1}\left[l_{i^{\prime}} b_{i^{\prime}}(x) \Gamma\left(\beta_{i^{\prime}}\right)\right]}{\Gamma\left(\sum_{i=1}^{j+1} \beta_{i^{\prime}}\right)} \int_{0}^{x}(x-s)^{\sum_{i=1}^{j+1} \beta_{i^{\prime}}-1} v(s) \mathrm{d} s
\end{aligned}
$$

This implies that the inequality (17) holds for $k=j+1$. Hence, it holds for any $k \in N$.

Since $b_{i}(i=1,2, \cdots, n)$ are bounded, $b_{i}<M_{i}\left(M_{i}>0\right)(i=1,2, \cdots, n)$ :

$$
\begin{aligned}
B^{k} v(x) \leq & \sum_{1^{\prime}, 2^{\prime}, \cdots \cdot k^{\prime}=1}^{n} \frac{\left[l_{1^{\prime}} b_{1^{\prime}}(t) \Gamma\left(\beta_{1^{\prime}}\right)\right]\left[b_{2^{\prime}}(x) \Gamma\left(\beta_{2^{\prime}}\right)\right] \cdots\left[b_{k^{\prime}}(x) \Gamma\left(\beta_{k^{\prime}}\right)\right]}{\Gamma\left(\beta_{1^{\prime}}+\beta_{2^{\prime}}+\cdots+\beta_{k^{\prime}}\right)} . \\
& \int_{0}^{x}(x-t)^{\sum_{i=1}^{k} \beta_{i^{\prime}}-1} v(t) \mathrm{d} t \\
\leq & \sum_{1^{\prime}, 2^{\prime}, \cdots \cdot k^{\prime}=1}^{n} \frac{\left(l_{1^{\prime}} l_{2^{\prime}} \cdots l_{k^{\prime}}\right)\left(M_{1^{\prime}} M_{2^{\prime}} \cdots M_{k^{\prime}}\right) \cdot\left(\Gamma\left(\beta_{1^{\prime}}\right) \Gamma\left(\beta_{2^{\prime}}\right) \cdots \Gamma\left(\beta_{k^{\prime}}\right)\right) .}{\Gamma\left(\beta_{1^{\prime}}+\beta_{2^{\prime}}+\cdots+\beta_{k^{\prime}}\right)} . \\
& \int_{0}^{x}(x-t)^{\sum_{i=1}^{k} \beta_{i^{\prime}}-1} v(t) \mathrm{d} t
\end{aligned}
$$

and according to the property of the Gamma function, $B^{k} v(x) \rightarrow 0$ as $n \rightarrow \infty$ for $x \in[0, T)$, then:

$$
\begin{aligned}
v(x) \leq & \tilde{a}(x)+\sum_{k=1}^{\infty}\left(\sum_{1^{\prime}, 2^{\prime}, \cdots k^{\prime}=1}^{n}\left(\frac{1}{p} b^{\frac{1-p}{p}}\right)^{k} \cdot \frac{\prod_{i=1}^{k}\left[l_{i^{\prime}} b_{i^{\prime}}(x) \Gamma\left(\beta_{i^{\prime}}\right)\right]}{\Gamma\left(\sum_{i=1}^{k} \beta_{i^{\prime}}\right)} .\right. \\
& \left.\int_{0}^{x}(x-t)^{\sum_{i=1}^{k} \beta_{i^{\prime}}-1} \tilde{a}(t) \mathrm{d} t\right) .
\end{aligned}
$$

Hence:

$$
\begin{aligned}
u(x) \leq & \left(\tilde{a}(x)+\sum_{k=1}^{\infty}\left(\sum_{1^{\prime}, 2^{\prime}, \cdots k^{\prime}=1}^{n}\left(\frac{1}{p} b^{\frac{1-p}{p}}\right)^{k} \cdot \frac{\prod_{i=1}^{k}\left[l_{l^{\prime}} b_{i^{\prime}}(x) \Gamma\left(\beta_{i^{\prime}}\right)\right]}{\Gamma\left(\sum_{i=1}^{k} \beta_{i^{\prime}}\right)} .\right.\right. \\
& \left.\left.\int_{0}^{x}(x-t)^{\sum_{i=1}^{k} \beta_{i^{\prime}}-1} \tilde{a}(t) \mathrm{d} t\right)\right)^{\frac{1}{p}}
\end{aligned}
$$

This completes the proof.

By Theorem 1, the main result in [16] is a special case of Theorem 1 for $p=1, f_{i}(t, u(t))=u(t)$.

Corollary 1. (see [16]). For any $t \in[0, T)$ :

$$
u(t) \leq a(t)+\sum_{i=1}^{n} b_{i}(t) \int_{0}^{t}(t-s)^{\beta_{i}-1} u(s) \mathrm{d} s
$$

where all the functions are non-negative and continuous. The constants $\beta_{i}>0, b_{i}(i=1,2, \cdots, n)$ are the bounded and monotonic increasing functions on $[0, T)$. Then:

$$
u(t) \leq a(t)+\sum_{k=1}^{\infty}\left(\sum_{1^{\prime}, 2^{\prime}, \ldots k^{\prime}=1}^{n} \frac{\prod_{i=1}^{k}\left[b_{i^{\prime}}(t) \Gamma\left(\beta_{i^{\prime}}\right)\right]}{\Gamma\left(\sum_{i=1}^{k} \beta_{i^{\prime}}\right)} \int_{0}^{t}(t-s)^{\sum_{i=1}^{k} \beta_{i^{\prime}}-1} a(s) \mathrm{d} s\right)
$$


Corollary 2. Under the hypothesis of Theorem 1, if a $(x)$ is increasing on $[0 . T]$, then:

$$
\begin{aligned}
u(x) & \leq\left(\tilde { a } ( T ) \sum _ { k = 0 } ^ { \infty } \left(\sum_{1^{\prime}, 2^{\prime}, \cdots k^{\prime}=1}^{n}\left(\frac{1}{p} b^{\frac{1-p}{p}}\right)^{k} .\right.\right. \\
& \frac{\prod_{i=1}^{k}\left[l_{i^{\prime}} b_{i^{\prime}}(x) \Gamma\left(\beta_{i^{\prime}}\right)\right]}{\Gamma\left(\sum_{i=1}^{k} \beta_{i^{\prime}}+1\right)} \cdot T^{\left.\left.\sum_{i=1}^{k} \beta_{i^{\prime}}\right)\right)^{\frac{1}{p}}}
\end{aligned}
$$

Proof. Since $a(x)$ is increasing, $\tilde{a}(x)$ is also increasing:

$$
\begin{aligned}
u(x) \leq & \left(\tilde{a}(x)+\sum_{k=1}^{\infty}\left(\sum_{1^{\prime}, 2^{\prime}, \cdots k^{\prime}=1}^{n}\left(\frac{1}{p} b^{\frac{1-p}{p}}\right)^{k} \cdot \frac{\prod_{i=1}^{k}\left[l_{i^{\prime}} b_{i^{\prime}}(x) \Gamma\left(\beta_{i^{\prime}}\right)\right]}{\Gamma\left(\sum_{i=1}^{k} \beta_{i^{\prime}}\right)} .\right.\right. \\
& \left.\left.\int_{0}^{x}(x-t)^{\sum_{i=1}^{k} \beta_{i^{\prime}}-1} \tilde{a}(t) \mathrm{d} t\right)\right)^{\frac{1}{p}} \\
\leq & \tilde{a}^{\frac{1}{p}}(x)\left(1+\sum_{k=1}^{\infty}\left(\sum_{1^{\prime}, 2^{\prime}, \cdots k^{\prime}=1}^{n}\left(\frac{1}{p} b^{\frac{1-p}{p}}\right)^{k} \cdot \frac{\prod_{i=1}^{k}\left[l_{i^{\prime}} b_{i^{\prime}}(x) \Gamma\left(\beta_{i^{\prime}}\right)\right]}{\Gamma\left(\sum_{i=1}^{k} \beta_{i^{\prime}}\right)} .\right.\right. \\
& \left.\left.\int_{0}^{x}(x-t)^{\sum_{i=1}^{k} \beta_{i^{\prime}}-1} \mathrm{~d} t\right)\right)^{\frac{1}{p}} \\
& \left(\tilde { a } ( x ) \sum _ { k = 0 } ^ { \infty } \left(\sum_{1^{\prime}, 2^{\prime}, \cdots k^{\prime}=1}^{n}\left(\frac{1}{p} b^{\frac{1-p}{p}}\right)^{k} \frac{\prod_{i=1}^{k}\left[l_{i^{\prime}} b_{i^{\prime}}(x) \Gamma\left(\beta_{i^{\prime}}\right)\right]}{\Gamma\left(\sum_{i=1}^{k} \beta_{i^{\prime}}+1\right)} \cdot t^{\left.\left.\sum_{i=1}^{k} \beta_{i^{\prime}}\right)\right)^{\frac{1}{p}}}\right.\right. \\
\leq & \left(\tilde{a}(T) \sum_{k=0}^{\infty}\left(\sum_{1^{\prime}, 2^{\prime}, \cdots k^{\prime}=1}^{n}\left(\frac{1}{p} b^{\frac{1-p}{p}}\right)^{k} \frac{\prod_{i=1}^{k}\left[l_{i^{\prime}} b_{i^{\prime}}(x) \Gamma\left(\beta_{i^{\prime}}\right)\right]}{\Gamma\left(\sum_{i=1}^{k} \beta_{i^{\prime}}+1\right)} \cdot T^{\sum_{i=1}^{k} \beta_{i^{\prime}}}\right)\right)^{\frac{1}{p}}
\end{aligned}
$$

The proof is completed.

Theorem 2. Under the conditions of Corollary 2, if $\omega_{i}(x)(i=1,2, \cdots, n)$ are bounded and monotonic increasing. $g_{i} \in C\left(R_{+} \times R_{+}, R_{+}\right)$with $0 \leq g_{i}(x, u)-g_{i}(x, v) \leq T_{i}(u-v)$ for $u \geq v \geq 0$, where $T_{i}$ is the Lipschitz constant. If the following inequality is satisfied:

$$
\begin{aligned}
u^{p}(x) \leq & a(x)+\sum_{i=1}^{n} \omega_{i}(x) \int_{0}^{x} h_{i}(t) g_{i}(t, u(t)) \mathrm{d} t \\
& +\sum_{i=1}^{n} b_{i}(x) \int_{0}^{x}(x-t)^{\beta_{i}-1} f_{i}(t, u(t)) \mathrm{d} t
\end{aligned}
$$

then:

$$
\begin{gathered}
u(x) \leq\left(E _ { n } \tilde { a } ( x ) \cdot \sum _ { k = 0 } ^ { \infty } \left(\sum_{1^{\prime}, 2^{\prime}, \cdots k^{\prime}=1}^{n}\left(\frac{1}{p} b^{\frac{1-p}{p}}\right)^{k} .\right.\right. \\
\frac{\prod_{i=1}^{k}\left[l_{i^{\prime}} b_{i^{\prime}}(x) \Gamma\left(\beta_{i^{\prime}}\right)\right]}{\Gamma\left(\sum_{i=1}^{k} \beta_{i^{\prime}}+1\right)} \cdot t^{\left.\left.\sum_{i=1}^{k} \beta_{i^{\prime}}\right)\right)^{\frac{1}{p}}}
\end{gathered}
$$

where $E_{i}(i=0,1, \cdots, n)$ is defined inductively as the composition of $i+1$ functional operators; i.e., $E_{i}=D_{i} D_{i-1} \cdots D_{0}$, where:

$$
\begin{aligned}
& D_{0} w=w \\
& D_{j} w=w+\left(E_{j-1} w_{j}\right)\left(\exp \int_{0}^{t} v_{j} E_{j-1} w_{j}\right) \int_{0}^{t} v_{j} w \mathrm{~d} s \quad(j=1,2, \cdots, n)
\end{aligned}
$$


Proof. Denote $u^{p}(x)=v(x), u(x)=v^{\frac{1}{p}}(x)$. Then:

$$
\begin{aligned}
v(x) \leq & a(x)+\sum_{i=1}^{n} b_{i}(x) \int_{0}^{x}(x-t)^{\beta_{i}-1} f_{i}\left(t, v^{\frac{1}{p}}(t)\right) \mathrm{d} t \\
& +\sum_{i=1}^{n} \omega_{i}(x) \int_{0}^{x} h_{i}(t) g_{i}\left(t, v^{\frac{1}{p}}(t)\right) \mathrm{d} t \\
\leq & a(x)+\sum_{i=1}^{n} b_{i}(x) \int_{0}^{x}(x-t)^{\beta_{i}-1} f_{i}\left(t, \frac{1}{p} b^{\frac{1-p}{p}} v(t)+\frac{p-1}{p} b^{\frac{1}{p}}\right) \mathrm{d} t \\
& +\sum_{i=1}^{n} \omega_{i}(x) \int_{0}^{x} h_{i}(t) g_{i}\left(t, \frac{1}{p} b^{\frac{1-p}{p}} v(t)+\frac{p-1}{p} b^{\frac{1}{p}}\right) \mathrm{d} t \\
\leq & a(x)+\sum_{i=1}^{n} b_{i}(x) \int_{0}^{x}(x-t)^{\beta_{i}-1}\left[f_{i}\left(t, \frac{1}{p} b^{\frac{1-p}{p}} v(t)+\frac{p-1}{p} b^{\frac{1}{p}}\right)\right. \\
& \left.-f_{i}\left(t, \frac{p-1}{p} b^{\frac{1}{p}}\right)+f_{i}\left(t, \frac{p-1}{p} b^{\frac{1}{p}}\right)\right] \mathrm{d} t+\sum_{i=1}^{n} \omega_{i}(x) \int_{0}^{x} h_{i}(t) . \\
& \left.g_{i}\left(t, \frac{1}{p} b^{\frac{1-p}{p}} v(t)+\frac{p-1}{p} b^{\frac{1}{p}}\right)-g_{i}\left(t, \frac{p-1}{p} b^{\frac{1}{p}}\right)+g_{i}\left(t, \frac{p-1}{p} b^{\frac{1}{p}}\right)\right] \mathrm{d} t \\
\leq & a(x)+\frac{1}{p} b^{\frac{1-p}{p}} \sum_{i=1}^{n} l_{i} b_{i}(x) \int_{0}^{x}(x-t)^{\beta_{i}-1} v(t) \mathrm{d} t \\
& +\sum_{i=1}^{n} b_{i}(x) \int_{0}^{x}(x-t)^{\beta_{i}-1} f_{i}\left(t, \frac{p-1}{p} b^{\frac{1}{p}}\right) \mathrm{d} t \\
& +\frac{1}{p} b^{\frac{1-p}{p}} \sum_{i=1}^{n} T_{i} \omega_{i}(x) \int_{0}^{x} h_{i}(t) v(t) \mathrm{d} t+\sum_{i=1}^{n} \omega_{i}(x) \int_{0}^{x} h_{i}(t) g_{i}\left(t, \frac{p-1}{p} b^{\frac{1}{p}}\right) \mathrm{d} t \\
= & \tilde{a}(x)+\frac{1}{p} b^{\frac{1-p}{p}} \sum_{i=1}^{n} l_{i} b_{i}(x) \int_{0}^{x}(x-t)^{\beta_{i}-1} v(t) \mathrm{d} t \\
& +\frac{1}{p} b^{\frac{1-p}{p}} \sum_{i=1}^{n} T_{i} \omega_{i}(x) \int_{0}^{x} h_{i}(t) v(t) \mathrm{d} t \\
&
\end{aligned}
$$

where:

$$
\begin{aligned}
\tilde{a}(x) & =a(x)+\sum_{i=1}^{n} b_{i}(x) \int_{0}^{x}(x-t)^{\beta_{i}-1} f_{i}\left(t, \frac{p-1}{p} b^{\frac{1}{p}}\right) \mathrm{d} t \\
& +\sum_{i=1}^{n} \omega_{i}(x) \int_{0}^{x} h_{i}(t) g_{i}\left(t, \frac{p-1}{p} b^{\frac{1}{p}}\right) \mathrm{d} t
\end{aligned}
$$

Let:

$$
z(x)=\tilde{a}(x)+\frac{1}{p} b^{\frac{1-p}{p}} \sum_{i=1}^{n} T_{i} \omega_{i}(x) \int_{0}^{x} h_{i}(t) v(t) \mathrm{d} t
$$

Then:

$$
v(x) \leq z(x)+\frac{1}{p} b^{\frac{1-p}{p}} \sum_{i=1}^{n} l_{i} b_{i}(x) \int_{0}^{x}(x-t)^{\beta_{i}-1} v(t) \mathrm{d} t
$$
we have:

By Inequality (37), we derive that $z(x)$ is nonnegative and increasing. According to Corollary 1 ,

$$
v(x) \leq z(x) \sum_{k=0}^{\infty}\left(\sum_{1^{\prime}, 2^{\prime}, \cdots k^{\prime}=1}^{n}\left(\frac{1}{p} b^{\frac{1-p}{p}}\right)^{k} \cdot \frac{\prod_{i=1}^{k}\left[l_{i^{\prime}} b_{i^{\prime}}(x) \Gamma\left(\beta_{i^{\prime}}\right)\right]}{\Gamma\left(\sum_{i=1}^{k} \beta_{i^{\prime}}+1\right)} \cdot x^{\sum_{i=1}^{k} \beta_{i^{\prime}}}\right)
$$


Combing (39) with (37):

$$
\begin{aligned}
& z(x) \leq \tilde{a}(x)+\frac{1}{p} b^{\frac{1-p}{p}} \sum_{i=1}^{n} T_{i} \omega_{i}(x) \int_{0}^{x} h_{i}(t) . \\
& \left(z(t) \sum_{k=0}^{\infty}\left(\sum_{1^{\prime}, 2^{\prime}, \cdots k^{\prime}=1}^{n}\left(\frac{1}{p} b^{\frac{1-p}{p}}\right)^{k} \cdot \frac{\prod_{i=1}^{k}\left[l_{i^{\prime}} b_{i^{\prime}}(t) \Gamma\left(\beta_{i^{\prime}}\right)\right]}{\Gamma\left(\sum_{i=1}^{k} \beta_{i^{\prime}}+1\right)} \cdot t^{\sum_{i=1}^{k} \beta_{i^{\prime}}}\right)\right) \mathrm{d} t \\
& \leq \tilde{a}(x)+\frac{1}{p} b^{\frac{1-p}{p}} \sum_{i=1}^{n} T_{i} \omega_{i}(x) \int_{0}^{x} \tilde{h}_{i}(t) z(t) \mathrm{d} t
\end{aligned}
$$

where:

$$
\tilde{h}_{i}(x)=h_{i}(t) \sum_{k=0}^{\infty}\left(\sum_{1^{\prime}, 2^{\prime}, \cdots k^{\prime}=1}^{n}\left(\frac{1}{p} b^{\frac{1-p}{p}}\right)^{k} \cdot \frac{\prod_{i=1}^{k}\left[l_{i^{\prime}} b_{i^{\prime}}(t) \Gamma\left(\beta_{i^{\prime}}\right)\right]}{\Gamma\left(\sum_{i=1}^{k} \beta_{i^{\prime}}+1\right)} \cdot t^{\sum_{i=1}^{k} \beta_{i^{\prime}}}\right)
$$

By Lemma 2, we obtain:

$$
z(x) \leq E_{n} \tilde{a}(x)
$$

By inequalities (39) and (42), we obtain (32). The proof is completed.

Corollary 3. Under the hypothesis of Theorem 2, if $p=1$, then:

$$
u(x) \leq E_{n} \tilde{a}(x) \cdot \sum_{k=0}^{\infty}\left(\sum_{1^{\prime}, 2^{\prime}, \cdots k^{\prime}=1}^{n} \frac{\prod_{i=1}^{k}\left[l_{i^{\prime}} b_{i^{\prime}}(x) \Gamma\left(\beta_{i^{\prime}}\right)\right]}{\Gamma\left(\sum_{i=1}^{k} \beta_{i^{\prime}}+1\right)} \cdot t^{\sum_{i=1}^{k} \beta_{i^{\prime}}}\right)
$$

\section{Applications}

In this section, we present two examples as applications of our results.

Example 1. The following initial value problems of fractional differential equation was considered in [16]:

$$
\begin{aligned}
& \sum_{i=1}^{n} D_{R}^{\beta_{i}} u(t)=f(t, u(t)), \\
& \left.\sum_{i=1}^{n} D_{R}^{1-\beta_{i}} u(t)\right|_{t=0}=\delta
\end{aligned}
$$

where $0<\beta_{1}<\beta_{2}<\cdots<\beta_{n}<1, t \in[0, T)$, f satisfies $|f(t, u(t))-f(t, v(t))| \leq l|u(t)-v(t)| ; l$ is the Lipschitz constant. $D_{R}^{\beta}$ and $I_{R}^{\beta}$ denote the Riemann-Liouville fractional derivative and fractional integral operators, respectively. The uniqueness of solutions was proved by Gronwall-Bellman inequality.

In this section, we study the Hyers-Ulam stability of this initial value problem.

As we know, if $u(t)$ is a solution of the differential Equation (44), then $u(t)$ satisfies the following integral equation:

$$
u(t)=I_{R}^{\beta_{n}} f(t, u(t))-\sum_{i=1}^{n-1} I_{R}^{\beta_{n}-\beta_{i}} u(t)-\delta \cdot \frac{t^{\beta_{n}-1}}{\Gamma\left(\beta_{n}\right)}
$$

Theorem 3. If $u_{\varepsilon}(t) \in C[0, T]$ satisfies:

$$
\left|\sum_{i=1}^{n} D_{R}^{\beta_{i}} u_{\varepsilon}(t)-f_{\varepsilon}\left(t, u_{\varepsilon}(t)\right)\right| \leq \varepsilon,
$$

then there exists a solution of (44) such that $\left|u(t)-u_{\varepsilon}(t)\right| \leq k \varepsilon$ for all $t \in[0, T]$. Where:

$$
k=\frac{\Gamma\left(\beta_{n}\right)}{\Gamma\left(\beta_{n}+1\right)}\left(1+\sum_{k=1}^{\infty} \sum_{1^{\prime}, 2^{\prime}, \cdots k^{\prime}=1}^{n} \frac{\prod_{i=1}^{k}\left[b_{i^{\prime}}(t) \Gamma\left(\beta_{i^{\prime}}\right)\right]}{\Gamma\left(\sum_{i=1}^{k} \beta_{i^{\prime}}+1\right)} \cdot T^{\sum_{i=1}^{k} \beta_{i^{\prime}}}\right.
$$


$b_{i^{\prime}}(t)=\frac{1}{\Gamma\left(\beta_{n}-\beta_{i}\right)}$.

Proof. According to (45), we know that $u_{\varepsilon}(t)$ satisfies:

$$
-I_{R}^{\beta_{n}} \varepsilon \leq u_{\varepsilon}(t)-\left[I_{R}^{\beta_{n}} f_{\varepsilon}\left(t, u_{\varepsilon}(t)\right)-\sum_{i=1}^{n-1} I_{R}^{\beta_{n}-\beta_{i}} u_{\varepsilon}(t)-\delta \cdot \frac{t^{\beta_{n}-1}}{\Gamma\left(\beta_{n}\right)}\right] \leq I_{R}^{\beta_{n}} \varepsilon
$$

Then:

$$
\begin{aligned}
\left|u(t)-u_{\varepsilon}(t)\right| \leq & \mid I_{R}^{\beta_{n}} f(t, u(t))-\sum_{i=1}^{n-1} I_{R}^{\beta_{n}-\beta_{i}} u(t) \\
& -I_{R}^{\beta_{n}} f_{\varepsilon}\left(t, u_{\varepsilon}(t)\right)+\sum_{i=1}^{n-1} I_{R}^{\beta_{n}-\beta_{i}} u_{\varepsilon}(t) \mid \\
& \leq I_{R}^{\beta_{n}} \varepsilon+\sum_{i=1}^{n-1} \frac{1}{\Gamma\left(\beta_{n}-\beta_{i}\right)} \int_{0}^{t}(t-s)^{\beta_{n}-\beta_{i}-1}\left|u(s)-u_{\varepsilon}(s)\right| \mathrm{d} s
\end{aligned}
$$

By Theorem 1, we have:

$$
\begin{aligned}
\left|u(t)-u_{\varepsilon}(t)\right| & \leq \varepsilon \frac{t_{n}^{\beta}}{\Gamma\left(\beta_{n}+1\right)}+\sum_{k=1}^{\infty} \sum_{1^{\prime}, 2^{\prime}, \ldots k^{\prime}=1}^{n} \frac{\prod_{i=1}^{k}\left[b_{i^{\prime}}(t) \Gamma\left(\beta_{i^{\prime}}\right)\right]}{\Gamma\left(\sum_{i=1}^{k} \beta_{i^{\prime}}\right)} . \\
& \int_{0}^{t}(t-s)^{\left.\sum_{i=1}^{k} \beta_{i^{\prime}}-1\right)} \cdot I_{R}^{\beta_{n}} \varepsilon \mathrm{d} s \\
& \leq \varepsilon \frac{\Gamma\left(\beta_{n}\right)}{\Gamma\left(\beta_{n}+1\right)}\left(1+\sum_{k=1}^{\infty} \sum_{1^{\prime}, 2^{\prime}, \ldots k^{\prime}=1}^{n} \frac{\prod_{i=1}^{k}\left[b_{i^{\prime}}(t) \Gamma\left(\beta_{i^{\prime}}\right)\right]}{\Gamma\left(\sum_{i=1}^{k} \beta_{i^{\prime}}+1\right)} \cdot T^{\left.\sum_{i=1}^{k} \beta_{i^{\prime}}\right)}\right.
\end{aligned}
$$

where $b_{i^{\prime}}(t)=\frac{1}{\Gamma\left(\beta_{n}-\beta_{i}\right)}$. This completes the proof.

Example 2. Consider the integral equation as follows:

$$
\begin{aligned}
u(x)= & u(0)+\sum_{i=1}^{n} \omega_{i}(x) \int_{0}^{x} h_{i}(t) g_{i}(t, u(t)) \mathrm{d} t \\
& +\sum_{i=1}^{n} b_{i}(x) \int_{0}^{x}(x-t)^{\beta_{i}-1} f_{i}(t, u(t)) \mathrm{d} t
\end{aligned}
$$

This includes the integer and fractional integral parts.

We assert the solution of this integral equation is bounded on $[0, T]$, provided $\omega_{i}, g_{i}, f_{i}(i=1,2, \cdots, n)$ satisfy the assumptions of Corollary 3.

Theorem 4. Let $u$ be a solution of (50) on $[0, T]$. If $\omega_{i}, g_{i}, f_{i}(i=1,2, \cdots, n)$ satisfy the assumptions of Corollary 7 , then for $x \in[0, T]$ :

$$
u(x) \leq E_{n} u(0) \cdot \sum_{k=0}^{\infty}\left(\sum_{1^{\prime}, 2^{\prime}, \ldots k^{\prime}=1}^{n} \frac{\prod_{i=1}^{k}\left[l_{i^{\prime}} b_{i^{\prime}}(x) \Gamma\left(\beta_{i^{\prime}}\right)\right]}{\Gamma\left(\sum_{i=1}^{k} \beta_{i^{\prime}}+1\right)} \cdot T^{\sum_{i=1}^{k} \beta_{i^{\prime}}}\right)
$$

Proof. If $u$ is a solution of (50), then by Corollary 3: 


$$
\begin{aligned}
|u(x)| \leq & |u(0)|+\sum_{i=1}^{n} \omega_{i}(x) \int_{0}^{x} h_{i}(t)\left|g_{i}(t, u(t))\right| \mathrm{d} t \\
& +\sum_{i=1}^{n} b_{i}(x) \int_{0}^{x}(x-t)^{\beta_{i}-1}\left|f_{i}(t, u(t))\right| \mathrm{d} t \\
& \leq E_{n} u(0) \cdot \sum_{k=0}^{\infty}\left(\sum_{1^{\prime}, 2^{\prime}, \cdots k^{\prime}=1}^{n} \frac{\prod_{i=1}^{k}\left[l_{i^{\prime}} i_{i^{\prime}}(x) \Gamma\left(\beta_{i^{\prime}}\right)\right]}{\Gamma\left(\sum_{i=1}^{k} \beta_{i^{\prime}}+1\right)} \cdot t^{\left.\sum_{i=1}^{k} \beta_{i^{\prime}}\right)}\right. \\
& \leq E_{n} u(0) \cdot \sum_{k=0}^{\infty}\left(\sum_{1^{\prime}, 2^{\prime}, \cdots k^{\prime}=1}^{n} \frac{\prod_{i=1}^{k}\left[l_{i^{\prime}} b_{i^{\prime}}(x) \Gamma\left(\beta_{i^{\prime}}\right)\right]}{\Gamma\left(\sum_{i=1}^{k} \beta_{i^{\prime}}+1\right)} \cdot T^{\left.\sum_{i=1}^{k} \beta_{i^{\prime}}\right)}\right.
\end{aligned}
$$

Remark 1. The result of Corollary 3 also can be used to prove the uniqueness of solutions to fractional differential equations.

Acknowledgments: This research is supported by the Natural Science Foundation of China (61473338) and the Doctoral Fund of Education Ministry of China (20134219120003).

Author Contributions: Yuqiang Feng introduced the problem and gave enough suggestions; Weimin Wang and Yuanyuan Wang investigated the problem; Weimin Wang wrote the paper.

Conflicts of Interest: The authors declare no conflict of interest.

\section{References}

1. Bihari, I. A generalization of a lemma of Bellman and its application to uniqueness problem of differential equation. Acta Math. Acad. Sci. Hung. 1956, 7, 81-94.

2. Chen, C.; Cheng, J.; Zhao, D. Gronwall-Bellman-Type integral inequalities and applications to BVPs. J. Inequal. Appl. 2009, 2009, 258569.

3. Dannan, F. Integral inequalities of Gronwall-Bellman-Bihari type and asymptotic behavior of certain second order nonlinear differential equations. J. Math. Anal. Appl. 1985, 108, 151-164.

4. Feng, Q.; Zheng, B. Generalized Gronwall-Bellman-type delay dynamic inequalities on time scales and their applications. Appl. Math. Comput. 2012, 218, 7880-7892.

5. Feng, Q.; Meng, F.; Zheng, B. Gronwall-Bellman type nonlinear delay integral inequalities on time scales. J. Math. Anal. Appl. 2011, 382, 772-784.

6. Ferreira, R.A.C.; Torres, D.F.M. Generalized retarded integral inequalities. Appl. Math. Lett. 2009, $22,876-881$.

7. Jung, S.-M. Hyers-Ulam-Rassias Stability of Functional Equations in Nonlinear Analysis; Springer Optimization and Its Applications; Springer: New York, NY, USA, 2011; Volume 48.

8. Kim, Y. Gronwall, Bellman and Pachpatte type integral inequalities with application. Nonlinear Anal. 2009, 27, e2641-e2656.

9. Li, L.; Meng, F.; Ju, P. Some new integral inequalities and their applications in studying the stability of nonlineari ntegro-differential equations with time delay. J. Math. Anal. Appl. 2010, 377, 853-862.

10. Liu, F.; Zhao, X. On the Hyers-Ulam stability of a innonhomogeneous linear differential equations of third-order. Math. Pract. Theory 2013, 43, 290-294.

11. Agarwal, R.; Deng, S.; Zhang, W. Generalization of a retarded Gronwall-like inequality and its applications. Appl. Math. Comput. 2005, 165, 599-612.

12. Cal, C.G.; Medjo, T.T. On a regularized family of models for homogeneous incompressiable two-phase flows. J. Nolinear Sci. 2014, 24, 1033-1103.

13. Ding, W.; Feng, P. The study of uniqueness of the steady state of the high degrees nonlinear nonautonomous circuits by Gronwall's Lemma. J. Huaiyin Inst. Technol. 2002, 11, 12-14. (In Chinese) 
14. Chorlay, R. From problems to structures: The cousin problems and the emergence of the sheaf concept. Arch. Hist. Exact. Sci. 2010, 64, 1-73.

15. Willett, D. A linear generalization of Gronwall's inequality. Proc. Am. Math. Soc. 1965, 16, 774-778.

16. Lin, S. Generalized Gronwall inequalitilies and their applications to fractional differential equations. J. Inequal. Appl. 2013, 2013, 549-558.

17. Jiang, F.; Meng, F. Explicit bounds on some new nonlinear integral inequality with delay. J. Comput. Appl. Math. 2007, 205, 479-486.

(C) 2017 by the authors. Licensee MDPI, Basel, Switzerland. This article is an open access article distributed under the terms and conditions of the Creative Commons Attribution (CC BY) license (http:/ / creativecommons.org/licenses/by/4.0/). 